\title{
Optical Response of Fluorescent Molecules Studied by Synthetic Femtosecond Laser Pulses
}

\author{
Arkaprabha Konar, ${ }^{\dagger}$ Jay D. Shah,,$^{\dagger}$ Vadim V. Lozovoy, ${ }^{\dagger}$ and Marcos Dantus ${ }^{*},, \neq$ \\ ${ }^{\dagger}$ Department of Chemistry, Michigan State University, East Lansing, Michigan 48824, United States \\ ${ }^{*}$ Department of Physics and Astronomy, Michigan State University, East Lansing, Michigan 48824, United States
}

Supporting Information

ABSTRACT: The optical response of the fluorescent molecule IR144 in solution is probed by pairs of collinear pulses with intensity just above the linear dependence using two different pulse shaping methods. The first approach mimics a Michelson interferometer, while the second approach, known as multiple independent comb shaping (MICS), eliminates spectral interference. The comparison of interfering and noninterfering pulses reveals that linear interference between the pulses leads to the loss of experimental information at early delay times. In both cases, the delay between the pulses is controlled with attosecond resolution and the sample fluorescence and stimulated emission are monitored simultaneously. An out-of-phase behavior is observed for fluorescence and stimulated emission, with the fluorescence signal having a minimum at zero time delay. Experimental findings are modeled using a two-level system with relaxation that closely matches the phase difference between fluorescence and stimulated emission and the relative intensities of the measured effects.

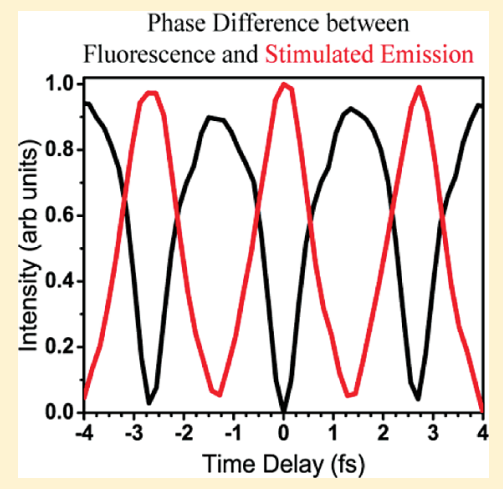

SECTION: Spectroscopy, Photochemistry, and Excited States

$\mathrm{T}$ he optical response of molecules in solution to a pulse of light has been the subject of numerous studies that have evolved with technology from the microsecond to the femtosecond time-scale. When femtosecond laser pulses are temporally overlapped, it is difficult to isolate the molecular response from pulse interference effects. This general difficulty has often been mitigated by ignoring the early time dynamics, while the pulses are overlapped, or by subtracting the early response as generated by the pure solvent. For example, when two collinear identical pulses, such as those generated by a Michelson interferometer, are scanned in time, they optically interfere, causing different amplitude modulation of the laser spectrum at different delay times. This interference may overwhelm the molecular dynamics that one wishes to measure. This simple fact has inspired experimental setups capable of measuring the early optical response of molecules with nearly identical pulses that do not interfere. One such setup, multiple independent comb shaping (MICS), ${ }^{1}$ introduced by Pestov, Lozovoy, and Dantus, is used here to study a model system, IR144, chosen due to its well-known fluorescent properties in solution. This letter compares the two experimental approaches (Michelson and MICS) and illustrates how MICS is able to obtain the expected molecular behavior without imposing spectral interference.

Wave-packet interferometry, demonstrated by Scherer et $\mathrm{al}^{2}$ used a pair of phase-locked femtosecond pulses to create vibrational wave packets in iodine. The intensity of the total fluorescence was measured as a function of delay between the two phase-locked pulses. Their experiment added a new dimension to coherent spectroscopy, leading to subsequent investigations $^{3}$ of the role of electronic and vibrational coherence versus the role of phase in atomic and molecular excitation. $^{4-8}$

Pulse sequence including replica pulses and pulse trains have been generated using pulse shapers for the past decade. ${ }^{10,11}$ The pulse shaper offers an all-optical setup, akin to a common path interferometer, with no mechanical moving parts, offering exceptional precision for a collinear geometry while eliminating the need of feedback stabilization. Phase and amplitude shaping, based on the time-to-frequency Fourier transform of the pulse replica, has been used conventionally to generate pulse pairs and pulse trains. ${ }^{11,12}$ For example, this approach was used by Hornung et al. ${ }^{13}$ to control the vibrational wave packet motion in the electronic ground and excited states of potassium and to study the influence of phase and amplitude modulated pulses on the molecular four-wave mixing response. Phase and amplitude modulation has also been mimicked by taking advantage of diffraction in a phase-only shaper. ${ }^{14}$ Zanni and coworkers have demonstrated the collection of $2 \mathrm{D}$ electronic spectra by means of pulse pairs generated using liquid crystalbased phase and amplitude shapers ${ }^{15}$ and in the mid-IR region using a 4-f acousto-optic modulator (AOM)-based polarization shaper. ${ }^{16}$ Phase-only generation of pulse trains has also been demonstrated by Weiner et al. ${ }^{17-19}$ Hildner et al have used phase-locked pulse pairs to study the vibrational and electronic coherences of single molecules embedded in a matrix. ${ }^{20,21}$

Received: March 23, 2012

Accepted: April 30, 2012 

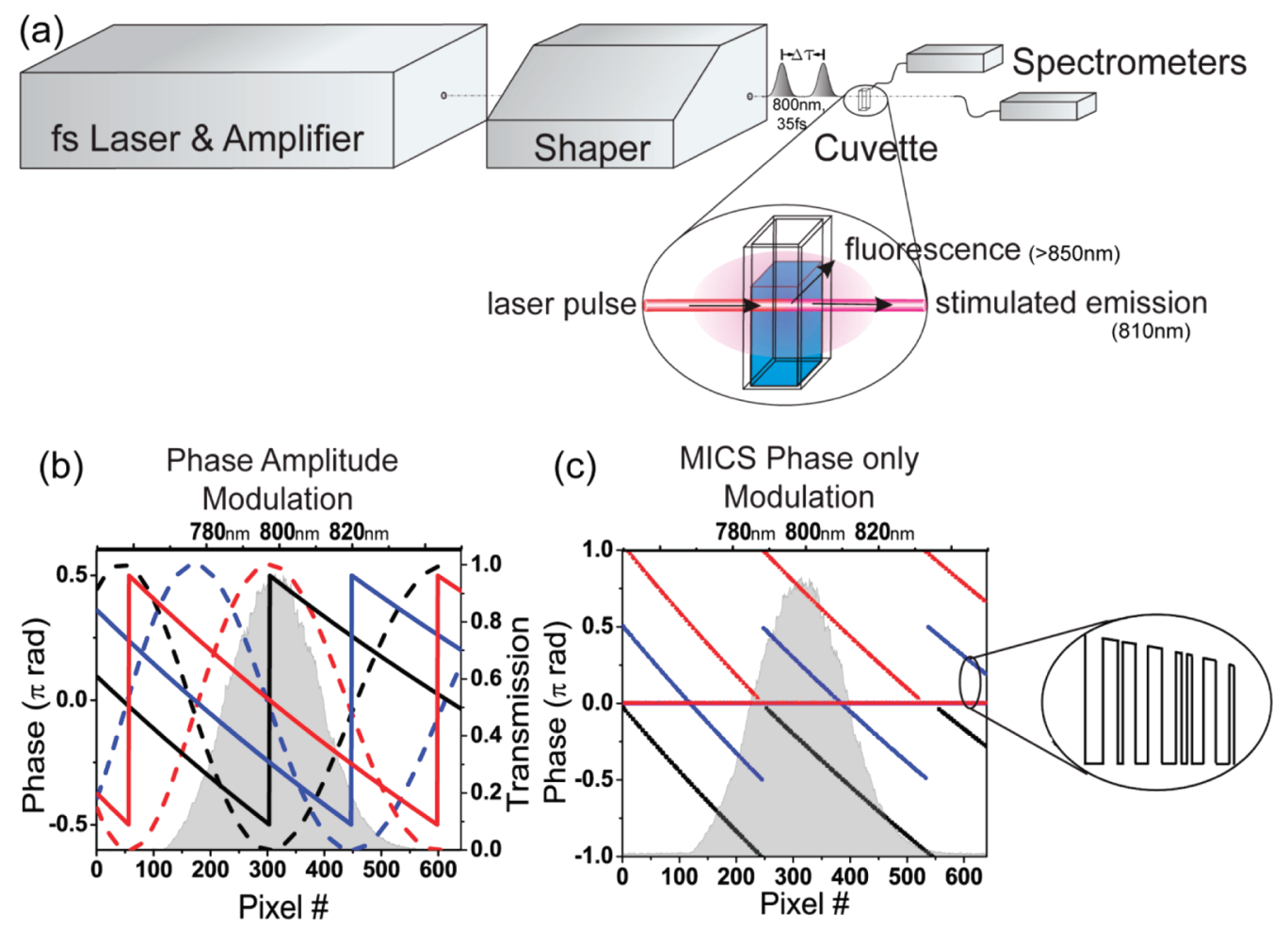

Figure 1. Experimental setup and two different pulse shaper parameters used to create interfering and noninterfering pairs of pulses. (a) Experimental setup for collection of fluorescence at right angles and stimulated emission from the sample. (b) Phase (solid) and amplitude (dashed) mask corresponding to a pair of interfering pulses with delays of $25.27 \mathrm{fs}$ (black, out-of-phase), $25.935 \mathrm{fs}$ (blue, in-quadrature), and $26.6 \mathrm{fs}$ (red, in phase). (c) Phase mask corresponding to MICS scan with the same time delays as in panel b.

These approaches work well; however, the spectrum of the field changes as a function of time delay. Thus interferometric transients obtained within the region of temporal overlap of the pulses are dominated by these amplitude masking effects. This highlights the need for an approach to perform experiments in the region of temporal overlap with a pair of noninterfering pulses. The approach taken here to solve the problem of scanning pulses without imposing large amplitude variations while generating noninterfering carrier phase locked stabilized pulse replicas is MICS.

IR 144 has been the subject of various studies because of its high absorption cross section at $800 \mathrm{~nm}$ and its large solventdependent Stokes shift. Dynamic absorption methods were used to project the phase relationships between the coherent wave packet motions on the ground-state and excited-state potential-energy surfaces. ${ }^{22}$ Solvation dynamics measurements were made using three pulse photon echo and transient grating techniques. ${ }^{9}$ Pump-probe investigations performed by $\mathrm{Yu}$ et $\mathrm{al}^{23}$ focused on the solvatochromatic behavior of the cyanine dyes. Very recently, single quantum three-dimensional (3D) electronic spectroscopy using the gradient assisted photon echo methodology was employed to resolve a cross peak originating from high frequency vibronic modes. ${ }^{24,25}$ Coherence period resolved transient grating was also used to measure the solvation dynamics of IR $144 .^{26}$ Due to the prevalence of IR144 data in the literature, the fluorophore serves as an ideal model system for investigating the contributions of different experimental designs to early optical response.

The experiment reported here compares two different approaches to synthesize pairs of identical pulses delayed in time. The first approach mimics a Michelson interferometer using phase and amplitude modulation. The shaped pulses are generated by imposing a transfer function on the input field, which consists of a sinusoidal amplitude mask with phase steps and an additional linear phase mask. The phase steps together with sinusoidal amplitude modulation provide the correct Fourier amplitude and phase for two delayed pulses, while the additional linear phase shifts both pulses in the time domain to keep one pulse fixed at time zero. Examples of the phase and amplitude modulations required to generate pulses separated by $\sim 25$ fs are shown in Figure 1b. Representative transmission and phase masks for delay times corresponding to out of phase (black), in-quadrature (blue), and in phase (red) are shown. Note that the out-of-phase transmission mask blocks most of the laser spectrum, while the in-phase transmission mask transmits most of the spectrum.

MICS is used to generate noninterfering pulse replicas by addressing a pair of frequency combs using phase-only shaping. ${ }^{1}$ On the basis of the fact that the slope of a linear phase corresponds to a time delay of a pulse, one is able to delay the entire pulse, or one is able to delay a portion of the original pulse. In this case, our goal is to generate two pulses with identical pulse duration and carrier frequency. Therefore, we sample the available spectral bandwidth, dividing it into two independent combs. In our original work, ${ }^{1}$ we separated the pulses by assigning an equal number of pixels to each pulse, for example, odd pixels are left with a phase of slope zero, while even pixels are assigned a linear phase modulation with a slope equal to the time delay desired. Here, we assigned at random 1 , 2 , and 3 pixel-binning to the delayed pulse in order to minimize the peak intensity of satellite pulses originating from the resulting gaps in the spectrum. The phase used to create the pulse pair using MICS, with phase masks corresponding to outof-phase (black), in-quadrature (blue), and in-phase (red) delay 


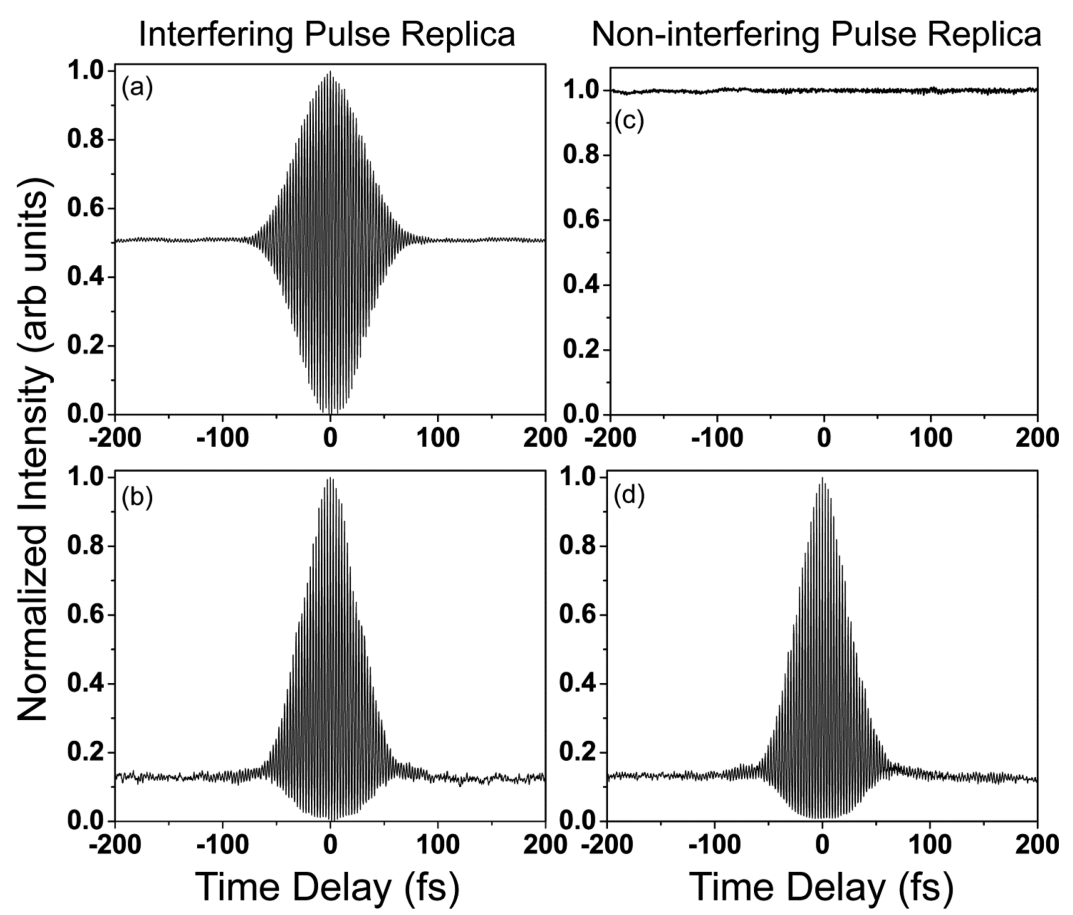

Figure 2. Experimental data: Interferometric (PA) time delay scans for the (a) laser intensity and (b) SHG intensity. Noninterfering (MICS) time delay scans for the (c) laser intensity and (d) SHG intensity.

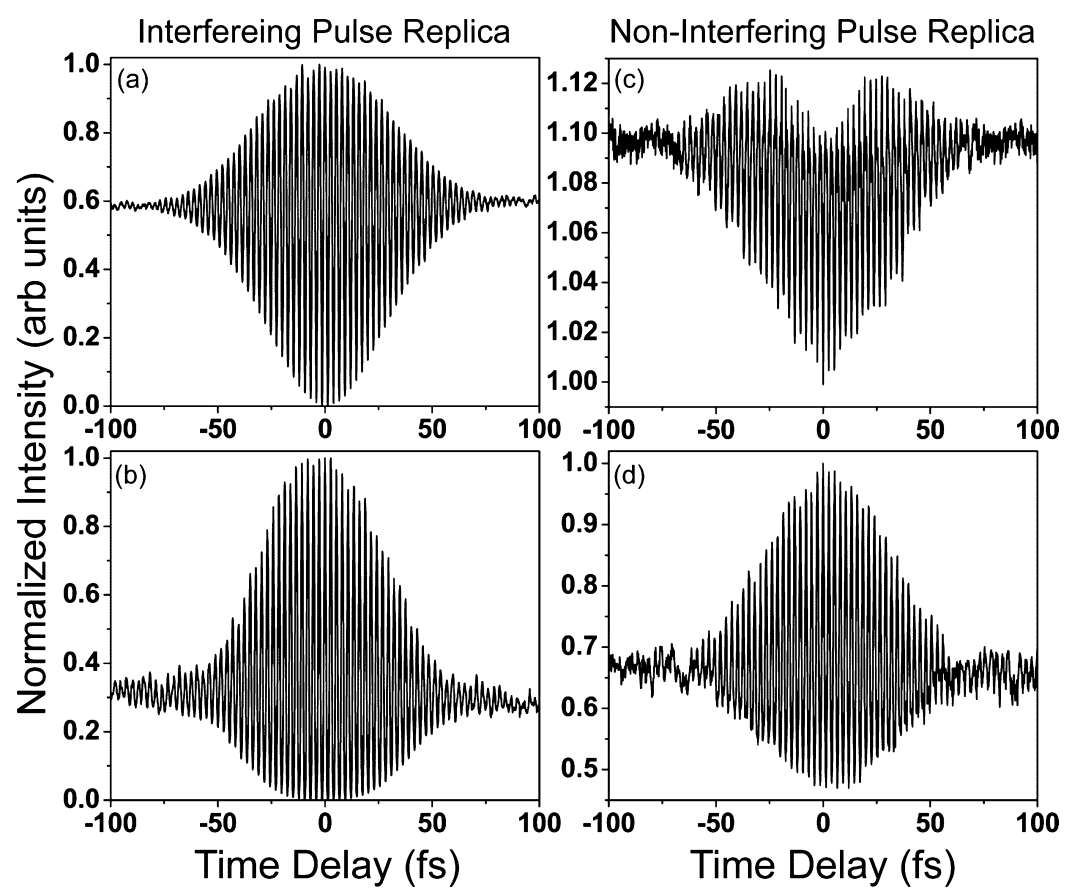

Figure 3. Experimental results: (a) Integrated fluorescence and (b) stimulated emission as a function of time delay using phase and amplitude modulation. (c) Integrated fluorescence and (d) stimulated emission as a function of time delay using MICS phase modulation.

times are shown in Figure 1c. The alternating and random pixel-width assignment of the phase is illustrated in the zoomed-in region of Figure 1c, where the thinnest column represents a single pixel. This approach helps in reducing and spreading the satellite pulses created at long delays (Supporting Information, Figure 3 ). The maximum time delay range, provided by the shaper with minimal diffractive loss, is $\pm 1.4 \mathrm{ps}$, and the smallest step size used for these experiments is 60 attoseconds. The single beam geometry, without beam splitters and optical delay lines, ensured outstanding stability, which is better than what can be achieved with an optical mechanical interferometer.

The experimental setup is described in the Experimental Methods section, and a sketch of the experimental setup is shown in Figure 1a. Both methods for creating pulse pairs were first tested by measuring the intensity of the modulated pulses as a function of time delay as shown in Figure 2a,c. Both scans were normalized to unity for transform limited pulses $(t=0$ for 
(a)

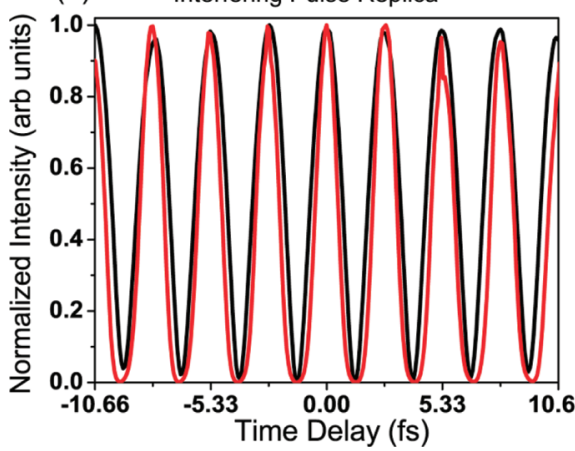

(b) Non-Interfering Pulse Replica

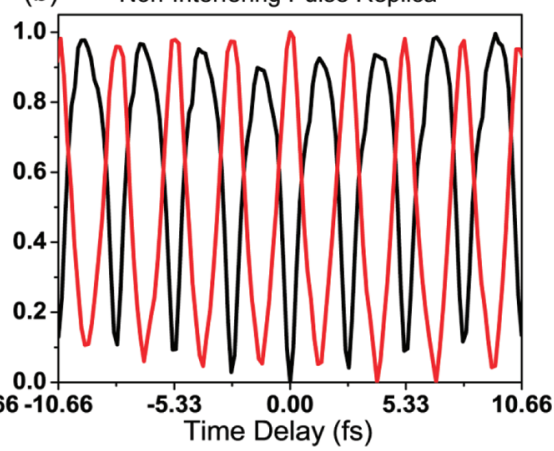

Figure 4. Comparison of the normalized stimulated emission (red) and fluorescence (black) signals when using (a) phase and amplitude and (b) MICS (phase only) modulation to generate delayed pulses. Note that fluorescence and stimulated emission signals are out-of-phase for MICS.

both methods). Note that the phase-amplitude modulation scan shows the expected interference between the two pulses at the optical frequency $\sim 2.66$ fs (Figure 2a). The interference subsides only after the pulses are no longer overlapped in time. The MICS scan shows little or no optical interference (Figure 2c), as desired. At long delay times, when using interfering pulses, half of the amplitude is lost because it is blocked by the amplitude mask. For MICS, half of the intensity of the pulses appears as broad satellites several picoseconds away from the central pulses (Supporting Information Figure 3). The second test of the methods involved collection of the second harmonic of the pulses when focused on a $100 \mu \mathrm{m}$ thick $\beta$-barium borate (BBO) crystal. The interferometric autocorrelation functions for each of the methods (PA and MICS) are shown in Figure $2 \mathrm{~b}, \mathrm{~d}$. Note the close agreement between the PA (phaseamplitude modulation) and MICS (pure phase modulation) methods. Note that second harmonic generation (SHG) autocorrelation, which involves sum-frequency mixing, leads to interference for MICS and is of comparable quality to that obtained by PA modulation. The time-domain explanation is that both methods produce similar pulse pairs, and the satellite pulses from MICS have little or no nonlinear optical contribution. Alternatively, the nonlinear optical interference is similar to that involved in multiphoton intrapulse interference. ${ }^{29,30}$ Intrapulse interference occurs when different frequency mixing paths can generate a particular frequency, for example $400 \mathrm{~nm}$. When all the paths have the same phase, constructive interference takes place, and a maximum is observed. Conversely, the phase of all the paths can be such that no $400 \mathrm{~nm}$ photons are generated.

The unfocused shaped laser pulses were then directed to a one-centimeter optical-path cell containing a $10^{-6} \mathrm{M}$ solution of IR144. The total integrated fluorescence (at right angles) and stimulated emission were collected as a function of delay time for both methods of pulse synthesis. The fluorescence and stimulated emission signals obtained when using interfering pulse replica are shown in Figure 3a,b, respectively. The graphs are normalized to unity for transform limited pulses. Interference fringes are observed at the optical frequency. The stimulated emission trace is very similar to the fluorescence trace. Note that both are very similar to the measured laser intensity as a function of time delay shown in Figure 2a. They are similar because the overall intensity of the shaped laser pulse is modulated by the linear interference (a laser effect) and not because of molecular properties. This is the effect we set out to mitigate through phase-only modulation.
Fluorescence and stimulated emission scans obtained as a function of time delay were recorded using noninterfering pulse replica based on MICS (Figure 3c,d), using the same peak power and dye concentration as for the other measurements. Fringes are again observed, with a periodicity corresponding to the optical frequency. The fluorescence intensity is found to be greater when the two pulses are out of phase with each other. A close look at the region near zero delay time (Figure $4 \mathrm{~b}$ ) shows that the fringes observed for fluorescence and for stimulated emission are exactly out of phase in contrast to the interfering pulse replica (Figure 4a). This modulation is observed because the experiments are carried out under slight saturation conditions, in a regime where there is a certain percent deviation from linearity. Under this condition, fluorescence is slightly depleted at time zero, while stimulated emission is maximized at zero delay. The fringes decay with a time scale comparable to the pulse duration, consistent with electronic dephasing occurring on a faster time scale than pulse duration.

The simplest theoretical model capable of reproducing the observed data is a two-level model with resonant frequency corresponding to the $S_{0}$ to $S_{1}$ absorption of IR144 in methanol without relaxation but with dephasing (Figure 5 inset). The

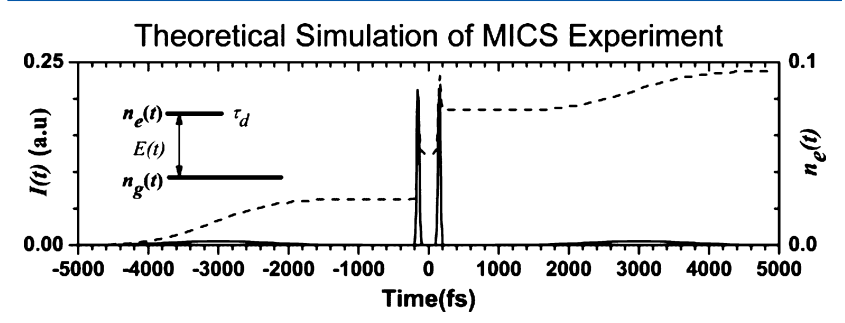

Figure 5. Theoretical simulation of the excited state population for a pair of pulses generated using MICS phase mask (dotted curve). The pair of pulses is separated by $300 \mathrm{fs}$ showing the satellite pulses at \pm 3 ps (solid curve).

dephasing rate was used as an adjustable parameter to achieve the best fit between experimental data and simulations. The electric field is modeled as a sum of two delayed pulses. In the case of MICS, modulation satellite pulses are placed at \pm 3 ps with energy equal to the difference between the total energy of the pulse minus the energy in the two delayed pulses, as shown in Figure 5. At time zero, all the energy is in the main pulse. For delay times shorter than the pulse duration, the intensity shuttles between the delayed pulses and the satellite peaks. At longer delay times, $50 \%$ of the intensity is found in the delayed pulses and 50\% in the satellite peaks. 


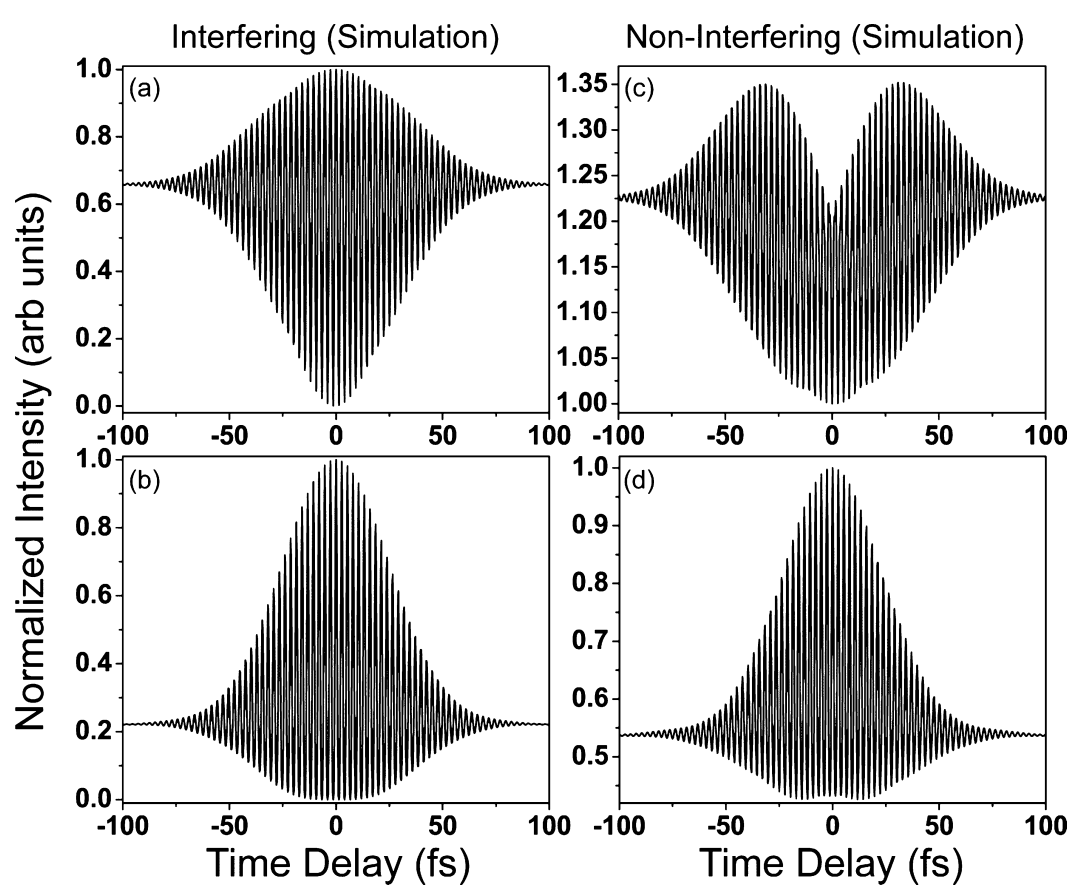

Figure 6. Simulation of the (a) integrated fluorescence and (b) stimulated emission signals as a function of time delay between the pulse pair generated using phase and amplitude modulation. (c) Integrated fluorescence and (d) stimulated emission signal as a function of time delay between the pulse pair generated using MICS phase mask.

Transitions between the ground and excited states are driven by the femtosecond laser pulses. The interaction of light with the two-level system described using Liouville equations are

$$
\begin{aligned}
\mathrm{d} \rho_{10}(t) / \mathrm{d} t= & -i \omega_{10} \rho_{10}(t)+i V(t) / \hbar\left[\rho_{11}(t)-\rho_{00}(t)\right] \\
& -\gamma \rho_{10}(t) \\
\mathrm{d} \rho_{11}(t) / \mathrm{d} t= & -i / \hbar\left[V(t) \rho_{10}(t)^{*}-\rho_{10}(t) V(t)^{*}\right]
\end{aligned}
$$

where $V(t)=\mu E(t) \exp \left(-i \omega_{0} t\right), \omega_{10}$, and $\omega_{0}$ are the resonant and the carrier frequencies; $\rho_{11}$ and $\rho_{00}$ are the diagonal elements of the density matrix of the two-level system describing populations in excited and ground states; $\rho_{10}$ and $\rho_{01}=\rho_{10} *$ are the off-diagonal elements of the density matrix describing electronic coherence between the two states; $\gamma$ is the electronic coherence dephasing rate. The measured fluorescence intensity is proportional to the excited state population $n_{\mathrm{e}}$ $=\rho_{11}$ after dephasing. The measured stimulated emission intensity is calculated in our model as an integral over time of the product of the excited state population, and the intensity of the pulse, $I(t) n_{\mathrm{e}}(t)$. The intensity of the laser pulse is varied to achieve best fitting of experimental effects and to have reasonable absorption probability. An example of the calculated population of excited states is presented in Figure 5, where a pair of pulses was generated using MICS phase shaping. The simulations show that the broad satellite pulses have an important contribution to the population in the excited state. In the weak field limit, both the satellite pulses and the short pulses contribute equally. Under saturation, the broad pulses have a greater contribution to the excited state population. This is due to the fact that there is a competition between dephasing of electronic coherence and stimulated transition from the excited state to the ground state. This also supports the experimentally observed minima in fluorescence and maxima in stimulated emission for short pulses when using MICS phases to delay the pulses.
Simulations of the fluorescence and stimulated emission measurements as a function of time delay for both pulse pair generation methods are shown in Figure 6. The dip in the fluorescence signal at early times was reproduced in the simulation by setting the dephasing time to $100 \mathrm{fs}$. A point worth noting is that the shape of the simulated curves does not depend very strictly on the two variable parameters (intensity and dephasing rate) in the model.

Here we have used two different methods to produce pairs of pulses for exploring the early optical response of IR144. In particular, we used MICS to explore how a pair of noninterfering pulses is able to probe molecular dynamics, while the pulses are temporally overlapped. Among the most important findings, we confirm that, under slight saturation conditions, fluorescence and stimulated emission are out of phase from each other. Maximum stimulated emission is found under conditions when fluorescence is minimal. Most importantly, this study shows a new approach to detecting nonlinear optical processes that are masked by linear interferometric effects, as found in conventional pump-probe experiments. The MICS method shown here identifies nonlinearity in a system that is resonant with the incident laser.

More elaborate methods for pulse synthesis may take advantage of these novel pulse shaping methodologies to further probe molecules. We have a study underway exploring the Stokes shift for IR144 and IR125 using shaped pulses. One could also envision using these pairs of pulses to probe other types of laser-matter interactions such as intense laser-molecule ionization and fragmentation, or laser machining. Similarly, one could envision more complicated pulse sequences aimed at extracting rephasing and nonrephasing dynamics such as those in photon echo experiments and more recently in phasemodulated two-dimesional (2D) fluorescence spectroscopy. ${ }^{31}$ 


\section{EXPERIMENTAL METHODS}

The femtosecond laser system used for this study consists of a regeneratively amplified Ti:Sapphire laser (Spitfire, SpectraPhysics) seeded by a $86 \mathrm{MHz}$ Ti:Sapphire oscillator (KM Laboratories) with a spectral bandwidth of $40 \mathrm{~nm}$ (fwhm) (Supporting Information, Figure 1). The output from the amplifier at $1 \mathrm{kHz}$ centered at $800 \mathrm{~nm}$ is $\sim 700 \mu \mathrm{J}$ and was attenuated before entering a phase-amplitude pulse shaper (MIIPS Box 640, Biophotonic Solutions). The dispersed spectrum covered 450 SLM pixels with a resolution of 0.2 $\mathrm{nm}$ per pixel. The output of the pulse shaper was frequency doubled by a $100 \mu \mathrm{m}$ thick BBO crystal at the sample plane, and the resulting second harmonic was spectrally filtered using an infrared cutoff filter (BG39). High-order phase distortions introduced by the optics in the laser system and setup are compensated by the multiphoton intrapulse interference phase scan (MIIPS) $)^{27,28}$ software resulting in $35 \mathrm{fs}$ transform limited (TL) pulses at the sample.

A $10^{-6} \mathrm{M}$ solution of IR 144 in methanol in a $1 \mathrm{~cm}$ cuvette was used as the sample. The sample was purchased from Exciton and used without further purification. The beam waist used (when intensity drops to $1 / \mathrm{e}^{2}$ ) is $2.7 \mathrm{~mm}$ and was measured using a beam profiler (Coherent).

\section{ASSOCIATED CONTENT}

\section{(5) Supporting Information}

A short discussion on the generation and control of the satellite pulses along with the experimental setup is presented. This material is available free of charge via the Internet at http:// pubs.acs.org

\section{AUTHOR INFORMATION}

\section{Corresponding Author}

*Address: Chemistry Building, 578 S. Shaw Lane, Michigan State University, East Lansing, MI 48824. E-mail: dantus@msu. edu.

\section{Notes}

The authors declare no competing financial interest.

\section{ACKNOWLEDGMENTS}

We thank DOE SISGR (DE-SC0002325), Dr. Jeff Krause, Program Manager, for support of this research. We also thank Drs. Dmitry Pestov and Haowen Li of Biophotonic Solutions, Inc. for generously sharing their pulse shaping expertise. M.D. would like to thank Professor G. R. Fleming for the thoughtful discussion on electronic coherence of large molecules in condensed phase.

\section{REFERENCES}

(1) Pestov, D.; Lozovoy, V. V.; Dantus, M. Multiple Independent Comb Shaping (MICS): Phase-Only Generation of Optical Pulse Sequences. Opt. Express 2009, 17, 14351-14361.

(2) Scherer, N. F.; Ruggiero, A. J.; Du, M.; Fleming, G. R. Time Resolved Dynamics of Isolated Molecular Systems Studies with Phase Locked Femtosecond Pulse Pairs. J. Chem. Phys. 1990, 93, 856-857.

(3) Scherer, N. F.; Carlson, R. J.; Matro, A.; Du, M.; Ruggiero, A. J.; Romerorochin, V.; Cina, J. A.; Fleming, G. R.; Rice, S. A. Fluorescence-Detected Wave Packet Interferometry - Time Resolved Molecular-Spectroscopy with Sequences of Femtosecond PhaseLocked Pulses. J. Chem. Phys. 1991, 95, 1487-1511.

(4) Scherer, N. F.; Matro, A.; Ziegler, L. D.; Du, M.; Carlson, R. J.; Cina, J. A.; Fleming, G. R. Fluorescence-Detected Wave Packet
Interferometry 2. Role of Rotations and Determination of the Susceptibility. J. Chem. Phys. 1992, 96, 4180-4194.

(5) Christian, J. F.; Broers, B.; Hoogenraad, J. H.; Vanderzande, W. J.; Noordam, L. D. Rubidium Electronic Wavepackets Probed by a Phase-Sensitive Pump-Probe Technique. Opt. Commun. 1993, 103, 79-84.

(6) Yeazell, J. A.; Raithel, G.; Marmet, L.; Held, H.; Walther, H. Observation of Wave Packet Motion Along Quasi-Landau Orbits. Phys. Rev. Lett. 1993, 70, 2884-2887.

(7) Ahn, J.; Weinacht, T. C.; Bucksbaum, P. H. Information Storage and Retrieval Through Quantum Phase. Science 2000, 287, 463-465.

(8) Monmayrant, A.; Chatel, B.; Girard, B. Quantum State Measurement Using Coherent Transients. Phys. Rev. Lett. 2006, 96, 103002.

(9) Joo, T. H.; Jia, Y. W.; Yu, J. Y.; Lang, M. J.; Fleming, G. R. ThirdOrder Nonlinear Time Domain Probes of Solvation Dynamics. J. Chem. Phys. 1996, 104, 6089-6108.

(10) Weiner, A. M.; Leaird, D. E. Generation of Terahertz-Rate Trains of Femtosecond Pulses by Phase-Only Filtering. Opt. Lett. 1990, 15, 51-53.

(11) Wefers, M. M.; Nelson, K. A. Programmable Phase and Amplitude Femtosecond Pulse Shaping. Opt. Lett. 1993, 18, 20322034.

(12) Galler, A.; Feurer, T. Pulse Shaper Assisted Short Laser Pulse Characterization. Appl. Phys. B: Lasers Opt. 2008, 90, 427-430.

(13) Hornung, T.; Meier, R.; de Vivie-Riedle, R.; Motzkus, M. Coherent Control of the Molecular Four-Wave-Mixing Response by Phase and Amplitude Shaped Pulses. Chem. Phys. 2001, 267, 261-276. (14) Vaughan, J. C.; Hornung, T.; Feurer, T.; Nelson, K. A. Diffraction-Based Femtosecond Pulse Shaping with a Two-Dimensional Spatial Light Modulator. Opt. Lett. 2005, 30, 323-325.

(15) Grumstrup, E. M.; Shim, S. H.; Montgomery, M. A.; Damrauer, N. H.; Zanni, M. T. Facile Collection of Two-Dimensional Electronic Spectra Using Femtosecond Pulse-Shaping Technology. Opt. Express 2007, 15, 16681-16689.

(16) Middleton, C. T.; Strasfeld, D. B.; Zanni, M. T. Polarization Shaping in the Mid-IR and Polarization-based Balanced Heterodyne Detection with Application to 2D IR Spectroscopy. Opt. Express 2009, 17, 14526-14533.

(17) Weiner, A. M.; Oudin, S.; Leaird, D. E.; Reitze, D. H. Shaping of Femtosecond Pulses Using Phase-Only Filters Designed by Simulated Annealing. J. Opt. Soc. Am. A 1993, 10, 1112-1120.

(18) Weiner, A. M. Femtosecond Pulse Shaping Using Spatial Light Modulators. Rev. Sci. Instrum. 2000, 71, 1929-1960.

(19) Weiner, A. M. Ultrafast Optical Pulse Shaping: A Tutorial Review. Opt. Commun. 2011, 284, 3669-3692.

(20) Brinks, D.; Stefani, F. D.; Kulzer, F.; Hildner, R.; Taminiau, T. H.; Avlasevich, Y.; Mullen, K.; van Hulst, N. F. Visualizing and Controlling Vibrational Wave Packets of Single Molecules. Nature 2010, 465, 905-U905

(21) Hildner, R.; Brinks, D.; Stefani, F. D.; van Hulst, N. F. Electronic Coherences and Vibrational Wave-Packets in Single Molecules Studied with Femtosecond Phase-Controlled Spectroscopy. Phys. Chem. Chem. Phys. 2011, 13, 1888-1894.

(22) Carson, E. A.; Diffey, W. M.; Shelly, K. R.; Lampa-Pastirk, S.; Dillman, K. L.; Schleicher, J. M.; Beck, W. F. Dynamic-Absorption Spectral Contours: Vibrational Phase-Dependent Resolution of LowFrequency Coherent Wave-Packet Motion of IR144 on the GroundState and Excited-State $\pi \rightarrow \pi^{*}$ Surfaces. J. Phys. Chem. A 2004, 108, 1489-1500.

(23) Yu, A. C.; Tolbert, C. A.; Farrow, D. A.; Jonas, D. M. Solvatochromism and Solvation Dynamics of Structurally Related Cyanine Dyes. J. Phys. Chem. A 2002, 106, 9407-9419.

(24) Fidler, A. F.; Harel, E.; Engel, G. S. Dissecting Hidden Couplings Using Fifth-Order Three-Dimensional Electronic Spectroscopy. J. Phys. Chem. Lett. 2010, 1, 2876-2880.

(25) Harel, E.; Fidler, A. F.; Engel, G. S. Single-Shot GradientAssisted Photon Echo Electronic Spectroscopy. J. Phys. Chem. A 2010, 115, 3787-3796. 
(26) Park, S.; Park, J.-S.; Joo, T. Solvation Dynamics by Coherence Period Resolved Transient Grating. J. Phys. Chem. A 2011, 115, 39733979.

(27) Coello, Y.; Lozovoy, V. V.; Gunaratne, T. C.; Xu, B. W.; Borukhovich, I.; Tseng, C. H.; Weinacht, T.; Dantus, M. J. Interference without an Interferometer: A Different Approach to Measuring, Compressing, and Shaping Ultrashort Laser Pulses. J. Opt. Soc. Am. B 2008, 25, A140-A150.

(28) Pastirk, I.; Resan, B.; Fry, A.; MacKay, J.; Dantus, M. No Loss Spectral Phase Correction and Arbitrary Phase Shaping of Regeneratively Amplified Femtosecond Pulses using MIIPS. Opt. Express 2006, 14, 9537-9543.

(29) Walowicz, K. A.; Pastirk, I.; Lozovoy, V. V.; Dantus, M. Multiphoton Intrapulse Interference. 1. Control of Multiphoton Processes in Condensed Phases. J. Phys. Chem. A 2002, 106, 93699373.

(30) Lozovoy, V. V.; Pastirk, I.; Walowicz, K. A.; Dantus, M. Multiphoton Intrapulse Interference. II. Control of Two and ThreePhoton Laser Induced Fluorescence with Shaped Pulses. J. Chem. Phys. 2003, 118, 3187-3196.

(31) Lott, G. A.; Perdomo-Ortiz, A.; Utterback, J. K.; Widom, J. R.; Aspuru-Guzik, A.; Marcus, A. H. Conformation of Self-Assembled Porphyrin Dimers in Liposome Vesicles by Phase-Modulation 2D Fluorescence Spectroscopy. Proc. Natl. Acad. Sci. U.S.A. 2011, 108, 16521 . 